\title{
COMPORTAMIENTO DE LOS PADRES Y AUTONOMIA DEL ADOLESCENTE
}

\section{PARENTAL BEHAVIOR AND ADOLESCENT AUTONOMY}

\section{AUTORES}

Dra. María José Criado del Pozo: Departamento de Educación mariajose.criado@uah.es

\section{CURRÍCULUM VITAE}

Profesora Departamento de Psicopedagogía y Ed. Física. Área de Psicología Evolutiva y de la Educación en la Escuela Cardenal Cisneros (Universidad de Alcalá de Henares) y autora de varios libros y artículos.

\section{RESUMEN}

El adolescente mientras construye su identidad está necesitado de modelos que le enseñen a solucionar los conflictos, a tomar decisiones y a asumir responsabilidades. Estos referentes son variados y pueden ser: el grupo de iguales, los medios de comunicación, los profesores y también los padres.

Por razones de espacio nos vamos a centrar en un contexto: el familiar, y más en concreto en los padres, para indicar aquellas pautas de comportamiento que ayudan más a que el adolescente aprenda las múltiples tareas que conforman la identidad.

\section{PALABRAS CLAVE}

Adolescente - Comportamiento - Autonomía - Desarrollo humano. 


\begin{abstract}
The teen's identity while building your models need to be taught to resolve conflicts, make decisions and take responsibility. These referents are varied and include: the peer group, media, teachers and parents.
\end{abstract}

For reasons of space we will focus in context: the family, and specifically in the parents, to indicate those behavior patterns that help further the teen learn the many tasks that make up the identity.

\title{
KEY WORDS
}

Teen - Behavior - Autonomy - Human development.

\section{ÍNDICE}

1. Introducción

2. Tareas con las que se enfrenta el adolescente

3. Objetivo que pretendemos

4. La individuación

5. El rol de los padres en el proceso de individuación

5.1 Los padres como guía

5.2 Poner control y reglas

5.3 Prestar cariño y afecto

5.4 Saber comunicarse

6. Emancipación familiar y prácticas de crianza

7. Bibliografía 


\section{Introducción}

El adolescente mientras construye su identidad está necesitado de modelos que le enseñen a solucionar los conflictos, a tomar decisiones y a asumir responsabilidades. Estos referentes son variados y pueden ser: el grupo de iguales, los medios de comunicación, los profesores y también los padres.

Por razones de espacio nos vamos a centrar en un contexto: el familiar, y más en concreto en los padres, para indicar aquellas pautas de comportamiento que ayudan más a que el adolescente aprenda las múltiples tareas que conforman la identidad.

\section{Tareas con las que se enfrenta el adolescente}

Una persona alcanza su identidad cuando ha adquirido un concepto claro sobre su propia persona. Cuando sabe responderse a sí mismo a las preguntas de quién soy yo y qué quiero yo, y además lo puede justificar con una serie de argumentos.

Erikson, que ha estudiado el desarrollo humano desde la primera infancia hasta la madurez como un proceso de génesis de identidad, como una tarea que dura toda la vida, considera que en la adolescencia esta tarea ocupa un lugar relevante. Ocurre que en este momento el desarrollo físico, las habilidades sociales y algunos ingredientes de naturaleza cognitiva maduran lo suficiente para poder acometer dicha tarea.

El concepto de identidad que defiende Erikson es psicosocial y supone la consecución de una serie de tareas relacionadas con a) la toma de decisiones ocupacionales y educacionales, tales como qué quiero ser yo de mayor, o en qué trabajo me encuentro mejor encajado; b) el conjunto de valores y de creencias, pudiendo contestar a preguntas como qué defiendo en religión, en política, o cuáles 
son mis modelos culturales; c) la opinión sobre sí mismo, relacionada con la aceptación de sí mismo y de su autoestima, la definición del rol sexual; d) el aspecto interpersonal, en cuyo apartado estaría el haber logrado la autonomía emocional, o lo que es lo mismo el haber alcanzado relaciones maduras respecto a los padres, otros adultos, y compañeros de igual edad y sexo distinto. Debido a que las tareas son numerosas y difíciles de conseguir es por lo que se necesita una moratoria o tiempo de aprendizaje. En este tiempo el adolescente experimenta, explora distintos campos, trata de encontrar algo que satisfaga sus necesidades, deseos y valores. Sin embargo la identidad lograda no está garantizada y en su lugar se pueden adoptar otras formas de identidad.(Criado del Pozo, 1996)

Hay factores que ayudan y otros que no ayudan a conseguir la identidad personal. Citaremos algunos de ellos: en primer lugar está el propio adolescente, con su historia pasada, su personalidad, su actitud, pues no es lo mismo estar pasivo que observar, analizar y reestructurar las opiniones y las ideas de los demás; en segundo lugar inciden los factores socio-económicos que pueden constreñir o ampliar su gama de posibilidades; por último influye el tipo de relación que establezca con los grupos sociales que le rodean: padres, amigos y profesores.

\section{Objetivo que pretendemos}

Como acabamos de ver las tareas que tiene que realizar el adolescente durante el periodo de la moratoria son múltiples y los factores que inciden variados. De ahí que forzosamente tengamos que reducir el campo de estudio y sea nuestro objetivo centrarnos en el análisis de una de las tareas, la adquisición de la autonomía emocional, haciendo una reflexión sobre el tipo de intervención por parte de los padres que sería más adecuada, ya que partimos del supuesto de que unas actuaciones tienen posiblemente unos efectos y otras otros. 
Ahora bien antes de seguir adelante es obligado preguntarnos qué tipo de persona adulta queremos formar para fomentar un tipo $\mathrm{u}$ otro de conductas.

Entendemos que el desarrollo debe estar dirigido a una cada vez mayor realización de los aspectos personales, sociales e interpersonales, tal y como lo expresan Zacarés y Serra, 1997, al indicar los siguientes tres parámetros básicos de madurez psicológica:a) AUTONOMIA: capacidad de funcionar completamente como individuo. b) RESPONSABILIDAD SOCIAL: Capacidad para funcionar competentemente como miembro de una sociedad. c) ADECUACION INTERPERSONAL: capacidad para funcionar competentemente a nivel interpersonal.

\section{La individuación}

Se denomina individuación al establecimiento de la autonomía psicológica respecto a los padres, con la sensación de que el adolescente tiene sus propias ideas, que no dependen de las ideas de los padres (Bos, 1962). Para que la individuación se lleve a cabo no es preciso que los adolescentes rechacen las normas y valores parentales, pues en realidad las diferencias en los valores entre los padres y los adolescentes no siempre son llamativas (Gallatin,1980). En este proceso de individuación, en síntesis, los pasos que sigue el adolescente son: primero se rebela y pone entre paréntesis lo recibido por los padres y en algunos casos después de pensar y analizar acepta los valores de sus padres como si fueran del propio adolescente. Parece ser que la buena comunicación que hayan mantenido desde la infancia los padres y los hijos es un buen reflejo del funcionamiento del sistema familiar que tanto ayuda a la individuación.

Sin embargo no todos los adolescentes consiguen la individuación. Unos, debido al aplazamiento cada vez más dilatado del acceso a la condición de adulto, causado por 
las dificultades sociales del trabajo y la vivienda, y también por las propias dificultades personales, continúan siendo eternos adolescentes que tienen sentimientos de inferioridad, incapacidad para tomar decisiones, pautas de comportamiento irresponsable, ansiedad y egocentrismo, Sebald (1976).

Otros adolescentes pueden estar desapegados emocionalmente de sus padres, pero no han conseguido ser autónomos y tener confianza en sí mismos. Esto suele ocurrir cuando los padres han impuesto estándares ideales, demasiado estrictos sin escuchar las razones que aduce el hijo. Ciertos casos de fugas de adolescentes o de consumo de drogas parecen seguir este patrón. En este caso el adolescente opta por una identidad negativa que se comprende como el intento de recuperar las riendas del destino de uno mismo. Debido a una falta de comunicación entre los padres y el hijo, éste pierde la autonomía y la iniciativa optando por la separación y oposición a los padres.

De lo expuesto se deduce que la separación emocional de los padres es buena, pero ¿hasta qué punto debe alcanzarse?. Lo fundamental no es la separación por la separación, sino lograr la confianza en uno mismo para pensar de manera autónoma, tomar decisiones y además ser responsable. La situación ideal es aquella en la que se encuentra un punto de equilibrio en el que el adolescente ni es dependiente de su familia, ni tiene separación total, está cercano a sus padres, es receptivo a sus necesidades y deseos, pero siendo una persona autónoma (Hoffman,1996).

\section{El rol de los padres en el proceso de individuación}

Las funciones que deben realizar los padres para ayudar al adolescente a conseguir su individuación no son privativas de este momento; su ejercicio ha de empezar en la infancia por ser el desarrollo un proceso continuo, cuyos objetivos deben empezar a alcanzarse desde el principio. Igual comportamiento ha de seguirse respecto al 
apartado de las prácticas de crianza que veremos más adelante. Las funciones son varias destacando las siguientes:

\subsection{Los padres como guía}

La familia es una organización que necesita de una conducción. Esta debe ser ejercida por los padres por ser personas adultas y responsables que tienen a su cargo y cuidado a seres en formación y no responsables de sí mismos. La función que ejerzan los padres debe ser de acompañar, de guiar a sus hijos, teniendo en cuenta que la cultura de cada época es diferente. Cambian las modas, las costumbres, las instituciones, las personas y sin embargo estos cambios deben de hacerlos compatibles con los valores y objetivos educativos. Lo que se está pidiendo a los padres es una adecuación al momento histórico y personal que vive el adolescente.

Ahora bien esta función de guía que tienen que realizar los padres deberá de ser compartida. Ambos, padre y madre han de guiar a sus hijos, para lo cual es indispensable el diálogo, la búsqueda de unificación de criterios dentro de la pareja, para evitar el riesgo de que las discrepancias se manifiesten en síntomas en el grupo familiar. Estas circunstancias pueden llevar a uno de los padres a construir alianzas con alguno de los hijos, cuando en realidad uno de los padres debe apoyarse en el reconocimiento del otro para tener un soporte firme.

En esta función de guía los padres aportan la experiencia y el conocimiento de la vida. Experiencia es el cúmulo de circunstancias que hemos ido viviendo, que han dejado una marca en la memoria ya sea de signo positivo o negativo. 
El conocimiento de la vida está asociado a la reflexión sobre las propias experiencias, sobre lo que hemos oído, visto o leído de la experiencia de los otros en cualquier parte del mundo y en cualquier tiempo. Ambas cosas, Experiencia y Conocimiento, configuran una particular visión de cada uno. Esta particular visión ha de entenderse como un bien que debe aprovecharse, sin embargo los padres tienen que pensar que parte de esta experiencia integrará el bagaje de los hijos, pero esa experiencia paterna deberá ser tamizada por la propia interpretación que los hijos hagan.

La transferencia de esta experiencia estará relacionada con el vínculo, el tipo de comunicación que se pueda establecer, además de las intenciones de los padres. No es igual que se transmita una experiencia o un conocimiento para mostrar la superioridad sobre la inhabilidad o inmadurez del hijo, o para coartar su incipiente independencia, que para mostrar cómo se resolvió una situación, comprendiendo que los hijos no aceptarán al pie de la letra nuestros consejos, que harán su propia experiencia y adquirirán su propio conocimiento. La actuación concreta de los padres se traduce en exponer su punto de vista ante una situación concreta, argumentar lo que están exponiendo, aplicarlo a la situación de su hijo, previendo las consecuencias que se pueden derivar. El hijo ha de reflexionar, subrayar las diferencias que encuentra, viendo si puede encontrar en lo que le están transmitiendo, algún aspecto que le pueda ser útil.

\subsection{Poner control y reglas}

El objetivo último que los padres deben proponerse para sus hijos es inculcarles autodisciplina que consiste en aprender a satisfacer las 
demandas de la vida en sociedad sin que los sujetos se resientan en el plano personal.

Hasta que llega el momento en el que la persona sabe qué es lo que tiene que hacer necesita normas que deben ser razonables y también esenciales (Devries,1995):

- Para la seguridad del adolescente, que debe aprender a evitar peligros.

- Para la armonía dentro de la familia

- Para la capacidad de entenderse y obtener beneficios en el instituto, así como para la autodisciplina requerida para alcanzar éxito en el campo laboral que elija

- Para su bienestar: Es preciso que aprenda reglas, roles, habilidades que desarrollen su autoestima y autocontrol

- Para el bienestar de la comunidad en que reside

Debido a que aprender las reglas es un proceso largo hay que ser constantes en esta acción. Si se repiten una y otra vez las consignas los hijos entienden estas cosas como elementos importantes y de peso en nuestras vidas.

Las normas también han de tener racionalidad, que estén bien fundamentadas y no sean una decisión arbitraria (Herbert 1992). Para ello se apelará al diálogo, a la persuasión y al consenso de la familia. Si esto es importante durante la infancia en este periodo debe tenerse más en cuenta. Los padres han de estar dispuestos a distinguir lo que es esencial y a escuchar las razones de sus hijos, aceptando en algunos casos las decisiones de los hijos en situación de provisionalidad para evaluar conjuntamente las consecuencias. 
Actitud complementaria a la firmeza es la Flexibilidad. Flexibilidad significa ceder en algunas circunstancias, lo cual no se interpreta como índice de blandura sino como elemento facilitador de unas relaciones en las que se da la confianza y la seguridad.

Es perjudicial que la exigencia entre en el terreno del amor propio, entonces pierde su sentido, pues no es una exigencia para educar al hijo sino que se utiliza para la propia autoafirmación, que en realidad es una pseudoafirmación.

Un chico exigido en extremo en las normas empezará a espiar la conducta de sus padres para encontrar un fallo y recriminarles esa contradicción en la conducta.

En otros casos el adolescente se someterá a la exigencia y crecerá con esa carga que le restará flexibilidad y calidez en sus actuaciones, no reconociéndose en el error que es la base de toda superación.

\subsection{Prestar cariño y afecto}

El amor es un sentimiento específico y esencial en la familia. Este afecto no está presente en otros microsistemas, tales como la escuela u otros. En la escuela el profesor tiene que ayudar al alumno pero el amor no tiene por qué sentirlo.

La necesidad de dar y recibir cariño debe estar presente a lo largo de todo el desarrollo, no siendo privativo de la infancia. El adolescente a lo largo de toda la etapa siente una gran demanda de afecto por parte de sus 
padres. Es posible que se muestre esquivo frente a algunas manifestaciones de cariño, especialmente ante la modalidad paternalista, pero aún entonces la necesidad sigue existiendo, aunque la apariencia desconcierte a los padres.

Cuánto más afecto haya entre padres e hijos, mayor atención pondrá el hijo en todo lo que los padres le digan. En este sentido es bueno expresarle en caso de disgusto que el motivo es la actitud concreta que sea y no es la descalificación total.

Relacionado con el amor está la solidaridad y el respaldo. La solidaridad se expresa en hechos cotidianos como la colaboración en las tareas del hogar, el cuidado del bien común y toda actitud donde se evidencien el reconocimiento del esfuerzo del otro, y el ofrecimiento de ayuda que podrá ser mayor o menor según las circunstancias.

Respaldar es proteger, guardar las espaldas, es lo que da seguridad en los momentos difíciles. El respaldo nos hace sentirnos acompañados, aunque estemos solos. El respaldo ayuda al adolescente a avanzar con más seguridad en las circunstancias de la vida.

Respaldar no es sinónimo de estar encima, es muchas veces la idea, la certeza de que los padres estarán presentes siempre que se los necesite. Este recurso a veces no se utiliza, pero es suficiente con tener la convicción de que está. Frente a un fracaso del tipo que sea amoroso, académico o profesional están los padres para apoyar, consolar, racionalizar la situación y empujar a seguir adelante, ofreciéndole estrategias cognitivas y emocionales para superar el fracaso y quitar dramatismo a las circunstancias. 


\subsection{Saber comunicarse}

Una buena comunicación familiar ayuda a los adolescentes a lograr un fortalecimiento psíquico. A través de la conversación el adolescente por una parte aprenderá a pensar por sí mismo y le ayudará a anticipar las consecuencias de sus acciones; y por otra parte podrá conocer la semejanza emocional con sus padres, cuando sus sentimientos se reflejan en los de sus padres y retornan a ellos. Aunque el adolescente tiene ya habilidades para sacar estas consecuencias es conveniente que sus padres demuestren en las situaciones concretas estos efectos.

Respecto al tema de la comunicación de los padres con los hijos es pertinente preguntarse qué se debe comunicar, cómo y cuándo. Los padres deben de dar ejemplo, compartiendo sus preocupaciones sin cargarlas de contenido angustioso, sus deseos, ambiciones etc. Con esta información el adolescente advierte que cada persona es un mundo de intereses e inquietudes particulares que deben de armonizarse.

Gracias a la comunicación el joven aprenderá a ponerse en el punto de vista de los otros, lo que le permitirá entender gestos y actitudes de los padres que sin la adecuada información pueden dar lugar a equívocos e interpretaciones erróneas. A la vez facilitará la posibilidad de que también él pueda verbalizar sus dificultades.

Un buen diálogo debe tener transparencia, algún gesto de complicidad, así como firmeza para dar una indicación. Es conveniente usar los mensajes "yo" en la afirmación de los sentimientos, en lugar de los mensajes "tú eres" que agregan a la personalidad del adolescente una descripción negativa. Ocurre que las cosas horribles que los padres dicen a sus hijos aquellos no las sienten, excepto en el momento de la pelea, y suelen tener un efecto 
perdurable. Muchos adolescentes se las creen, se las toman en serio y actúan de acuerdo con dichas "descripciones".

El emplear tonos imperativos y amenazas nos conducen a la incomunicación, el desinterés, el mal humor y el resentimiento.

Los mejores momentos para hablar están vinculados a la oportunidad, referida bien al momento que sale un tema determinado o aquel otro en el que el hijo tiene mayor capacidad de escucha, pues lo que se pretende es causar un efecto, dejar una marca, por eso debemos de tener la certeza de que lo que se quiere comunicar llega al hijo.

\section{Emancipación familiar y prácticas de crianza}

Las prácticas de crianza difieren de unas familias a otras, favoreciendo o no la autonomía de sus hijos. Los distintos modos de disciplina parental se correlacionan con la probabilidad de rechazo de los padres autoritarios y la aceptación de los permisivos y de los democráticos.

Sin embargo aunque tanto los padres permisivos como los democráticos ayudan al adolescente a conseguir su individuación, hay diferencias entre ambos. Los padres permisivos exigen pocas responsabilidades; permiten a sus hijos que regulen sus propias actividades; evitan el ejercicio del control y no estimulan a sus hijos a que obedezcan normas que les llegan definidas desde fuera.

El equilibrio es conseguir adultos socialmente independientes, emocionalmente autónomos y también responsables, siendo Baumrind (1971) quien mejor lo expresa cuando habla del padre "autorizado" cuyos comportamientos son: Las situaciones de la vida cotidiana se dirigen de una manera racional; valora tanto la autoexpresión del 
adolescente como su respeto por la autoridad, el estudio y el trabajo; aprecia la voluntad independiente y la conformidad disciplinada; ejerce un firme control en los aspectos que hay divergencias pero no lo acorrala con constantes restricciones. Los padres con autoridad afirman las cualidades de sus hijos pero también le fijan modelos, criterios y consecuencias para su conducta futura.

Los efectos que produce en los hijos este tipo de interacción son el desarrollo de la competencia social, la adquisición de una ideología, la confianza en sí mismo y la responsabilidad.

\section{Bibliografía}

BAUMRIND,D. (1971): "Current patterns of parental authority", Developmental psychology monograph, 4 (1), part 2

BLOS,P. (1962): On adolescence: A psychoanalytic interpretation. Free Press, Nueva york

CRIADO DEL POZO, M.J. y otros (1997): "La personalidad adolescente". Actas VII Congreso INFAD, Oviedo

DEVRIES, R.A y otro. (1995): Adolescencia: Desafío para padres. Paidós

GALLATIN,J.(1980): "Political thinking in adolescence" en Adelson s. (ed): handbook of adolescent psychlogy, Wiley, Nueva York

HERBERT,M. (1992): Entre la tolerancia y la disciplina. Paidós

HOFFMAN,L. y otros (1996): Psicología del desarrollo hoy. volumen 2. Mc Graw Hill 
ROY HOPKINNS,J.(1987): Adolescencia, años de transición. Pirámide

ZACARES, J.J Y SERRA, E. (1997):La madurez psicológica. Perspectivas desde la Psicología. Pirámide 